\title{
Desarrollo psicomotor y cortisol salival en niños que viven juntos a sus madres privadas de libertad
}

\author{
Psychomotor development and cortisol salivary levels in infants \\ that live with their inmate mothers
}

\author{
Gia Haquin Macari ${ }^{\mathrm{a}}$, Adriana Gallardo Tapia ${ }^{\mathrm{a}}$, German Iñiguez ${ }^{\mathrm{b}}$, Gerardo Weisstaub $^{\mathrm{c}}$
}

aBecada de Pediatría. Facultad de Medicina, Campus Centro, Universidad de Chile bInstituto de Investigaciones Materno Infantil (IDIMI), Facultad de Medicina, Campus Centro, Universidad de Chile 'Departamento de Pediatría, Campus Centro, Facultad de Medicina, Instituto de Nutrición y Tecnología de los Alimentos (INTA), Universidad de Chile

Recibido: 5 de abril de 2018; Aceptado: 15 de diciembre de 2018

\section{Resumen}

En Chile el sistema penitenciario cuenta con un programa que permite que las madres privadas de libertad vivan con sus hijos menores de 2 años. Esta modalidad podría implicar que los niños estén más expuestos a condiciones de estrés y a mayor riesgo de retraso en su desarrollo psicomotor (DSM). Objetivo: Comparar el DSM y la concentración de cortisol en saliva de los niños que viven en la cárcel junto a sus madres y comparar los resultados con los observados en niños que no están bajo este régimen. Sujetos y Método: Estudio transversal en 42 lactantes, 12 de ellos hijos de madres reclusas en el centro penitenciario de Santiago (CPF), y 30 controles provenientes de un Centro de Salud Familiar de Atención Primaria (CESFAM). Se evaluó DSM de los lactantes mediante la encuesta ASQ-3 y se realizó medición de cortisol salival mediante radioinmunoensayo a los lactantes y madres. Resultados: La mediana de cortisol salival de los hijos de madres del CPF y CESFAM fue de $2,3 \mathrm{ng} / \mathrm{ml}$ (IQR 1,1 a 2,7 ) y de $2,1 \mathrm{ng} / \mathrm{ml}$ (IQR 1,6 a 2,9 ) respectivamente. El cortisol materno fue $4,6 \mathrm{ng} / \mathrm{ml}$ (IQR 3,8 a 7,3) en el CPF y $3,7 \mathrm{ng} / \mathrm{ml}$ (IQR 2,4 a 4,7) en el CESFAM. El déficit del DSM fue 2,3\% y 28,5\% para los niños del CPF y del CESFAM, respectivamente, sin diferencia estadística $(p=0,06)$. Conclusiones: No hubo diferencia en el DSM y tampoco en el cortisol salival entre los niños de ambos grupos.
Palabras clave:

Desarrollo psicomotor; estrés; lactantes; cortisol; saliva; centro penitenciario; centro de salud familiar

Correspondencia:

Gerardo Weisstaub

gweiss@inta.uchile.cl 


\begin{abstract}
In Chile, the prison system has a program that allows inmate mothers to live with their children under two years of age. This could imply that these children are more exposed to stress conditions and a higher psychomotor developmental delay (PDD) risk. Objective: To compare the PDD and salivary cortisol concentrations (SCC) of children living in prison with their mothers and to compare the results with control children. Subjects and Method: Cross-sectional study in 42 infants, 12 of them are children of inmate mothers in the penitentiary center (CPF) of Santiago, and 30 controls from a Primary Care Family Health Center (CESFAM). PDD of infants was assessed through the ASQ-3 questionnaire and salivary cortisol was measured in infants and mothers using radioimmunoassay. Results: The median salivary cortisol level of the children of CPF and CESFAM mothers was $2.3 \mathrm{ng} /$ $\mathrm{ml}$ (IQR 1.1 to 2.7 ) and $2.1 \mathrm{ng} / \mathrm{ml}$ (IQR 1.6 to 2, 9) respectively. Maternal cortisol was $4.6 \mathrm{ng} / \mathrm{ml}$ (IQR 3.8 to 7.3 ) in the CPF and $3.7 \mathrm{ng} / \mathrm{ml}$ (IQR 2.4 to 4.7 ) in the CESFAM. The PDD deficit was $2.3 \%$ and $28.5 \%$ for children from the CPF and the CESFAM respectively, without statistical difference $(p=0.06)$. Conclusions: There was no difference in the PDD and salivary cortisol between children of both groups.
\end{abstract}

\section{Keywords:}

Psychomotor development; stress; infants; cortisol; saliva; penitentiary center; family health center

\section{Introducción}

El desarrollo psicomotor (DSM) es el conjunto de habilidades que el niño va logrando producto de la maduración del Sistema Nervioso Central y de la interacción con el medio, fijando las bases para el futuro aprendizaje de los niños ${ }^{1}$. La prevalencia estimada del retraso del DSM en niños chilenos atendidos en el sistema público de salud es más baja que la observada en los países industrializados ( $11 \%$ vs 12 a $16 \%$, respectivamente) $)^{2,3}$. Llama la atención que los datos del Departamento de Estadística nacional obtenidos de los Resúmenes Estadísticos Mensuales (REM) 2016, muestran que la prevalencia de retraso del DSM varía entre 2 y $7 \%$ en menores de 2 años ${ }^{4}$. Es posible que exista un sub registro relacionado con el uso de pruebas de screening del DSM utilizadas en nuestro país: Escala de Evaluación del Desarrollo Psicomotor (EEDP) y Test de Desarrollo Psicomotor (TEPSI).

En los últimos años se han desarrollado nuevos instrumentos de tamizaje basados en el reporte de padres o cuidadores principales, como por ejemplo el Ages and Stages Questionnaires (ASQ), el cual está siendo ampliamente utilizado en USA y otros países. Este instrumento ha sido validado con respecto a el método de Bayley con su versión en español también en Chile ${ }^{1,5,6}$. Ages and Stages Questionnaires ha mostrado una sensibilidad y especificidad del $73 \%$ y $81 \%$ respectivamente al compararlo con Bayley, test considerado gold standar.

Por otro lado, es sabido que hay distintos factores ambientales y sociales que pueden influir en el desarrollo de los niños, siendo uno de estos el estrés, teniendo efectos evidentes sobre el neurodesarrollo en los primeros años de vida ${ }^{7,8}$.

Una de las maneras de evaluar el grado de estrés es medir el cortisol salival, una hormona esteroidea producida en el eje hipotálamo-pituitario-adrenal (HPA) que se secreta en la saliva, en reacción al estrés, por lo cual, ha sido utilizado como biomarcador de éste en población adulta y pediátrica ${ }^{8}$. Hay numerosa evidencia que menciona que el uso de cortisol salival se correlaciona significativamente con el cortisol total

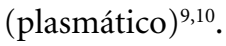

Estudios observacionales sugieren que el estrés de la vida temprana se asocia con déficits cognitivos y niveles elevados de cortisol en los niños ${ }^{11-13}$. Además, habrían evidencias que en niños pequeños que mantienen niveles de cortisol elevados muestran menor desempeño cognitivo ${ }^{14-16}$.

Dentro de otros factores ambientales que pueden influir en el neurodesarrollo se encuentra la privación de contacto materno, pobreza y otras condiciones sociales $^{17-19}$. La separación precoz, debido a la reclusión materna, corta el vínculo del apego y puede tener un impacto nocivo en un niño que se encuentra en desarrollo, el que ha sido descrito por algunos expertos como un "trauma perdurable"20-24.

Actualmente en Chile las mujeres representan el $10 \%$ de la población penal, $95 \%$ de las cuales son madres y $2 / 3$ tienen hijos menores de edad ${ }^{25,26}$. La vida en un centro penal es compleja, las mujeres que viven allí con sus hijos tienen que adaptarse a una disciplina muy rígida y muchas veces las condiciones de vida están lejos de ser las más adecuadas (ej: hacinamiento, falta de agua caliente y calefacción). En general se trata de mujeres jóvenes, que en su gran mayoría no han terminado la enseñanza media y han vivido toda su vida con privaciones. Las madres están expuestas frecuentemente a situaciones estresantes: no pueden acompañar a sus hijos al médico si estos se enferman, deben enfrentar el juicio y requisas, y muchas veces 
tienen una escasa red social de apoyo fuera del centro penitenciario ${ }^{27}$.

En este contexto, y bajo el principio de protección y promoción de los derechos del niño, en Chile se facilita el contacto entre madres internas y sus hijos en una sección del recinto penal, donde se permite que las madres vivan junto a éstos, o a ellos durante los primeros 2 años de vida ${ }^{28}$. Al mismo tiempo, en algunos centros como el centro penitenciario de San Joaquín existe además un programa de la fundación Integra que consiste en proveer de un sistema de sala cuna para los hijos de las internas. En la actualidad, no existen estudios que hagan un análisis respecto a la evaluación del desarrollo psicomotor de estos niños. El objetivo del estudio fue describir el DSM y la concentración de cortisol en saliva (CCS) de los niños que viven en la cárcel junto a sus madres y comparar los resultados con los observados en niños que no están bajo este régimen.

\section{Sujetos y Método}

\section{Diseño del estudio}

Estudio transversal, en el cual se incluyeron a todos los niños menores de 2 años, sin patología aguda, que vivían juntos a sus madres en el centro penitenciario femenino (CPF) de San Joaquín y a un grupo de niños, pareados por edad, inscritos en el Centro de Salud Familiar de Atención Primaria (CESFAM) "Padre Vicente Irarrázaval” de la comuna de Estación Central, perteneciente al sistema público de salud. Los criterios de exclusión fueron los niños que presentaron patologías que pudiesen influir negativamente en el DSM, hospitalizaciones superiores a 1 mes, y cuya madre no tenía dominio del idioma español.

Al inicio de la evaluación, se registró edad materna, tiempo de permanencia en CPF, patologías perinatales, consumo de drogas durante el embarazo, edad gestacional al momento del parto, presencia o ausencia de lactancia materna, escolaridad materna, y presencia de enfermedad aguda de la madre y/o hijo.

Se consideró como variables dependientes al DSM, niveles de cortisol salival en niños y como variables independientes al sexo, edad cronológica del niño, nivel de concentraciones de cortisol salival materno, estado nutricional del niño, nivel educacional alcanzado por la madre y edad materna.

\section{Herramientas utilizadas para medición}

\section{Desarrollo psicomotor}

Para evaluar DSM de los niños, se utilizó ASQ-3. Este test es un instrumento de screening para niños de 1 mes hasta los 5 años y que consta de 5 dominios (co- municación, motricidad fina, motricidad gruesa, resolución de problemas y relaciones interpersonales). Se ocupó el test correspondiente según edad cronológica de los niños y en el caso de prematuros se empleó la edad corregida. En cuanto a la aplicación del test cada niño fue evaluado en una sola oportunidad y su madre fue quién respondió a las preguntas. Se utilizó material accesorio explicativo y se ayudó a contestar el cuestionario cuando surgieron dudas de la madre respecto al hito alcanzado.

No hay consenso en la literatura sobre qué criterios ocupar para definición de déficit del desarrollo psicomotor, la mayoría de los autores recomienda basarse en los dominios, considerando déficit de DSM, los casos que tengan al menos un área descendida con un puntaje bajo -2DS del promedio. Esta propuesta fue la que nosotros consideramos para realizar nuestro estudio.

En Chile fueron validados los cuestionarios de 8, 18 y 30 meses. En un análisis preliminar se demostró que el uso de los valores de corte de la validación original de USA eran aplicables a nuestra población, por lo que para este estudio se utilizaron los puntos de corte publicados, considerando como riesgo de déficit del DSM tener al menos un área por debajo de los puntos de corte $^{29,30}$.

\section{Medición del cortisol}

El cortisol salival fue tomado a los niños y madres entre las 8:00 y 11:00 am del día. Las madres confirmaron no haber recibido alimento; ni ella ni su hijo, al menos una hora antes ${ }^{31}$, se les hizo salivar en una tórula de algodón y se recolectó la muestra en un tubo eppendorf, siendo almacenadas a $-20^{\circ} \mathrm{C}$ hasta ser procesadas en ciego. Se descongelaron, y centrifugaron por $5 \mathrm{~min}$ a $3.000 \mathrm{rpm}$ y el sobrenadante se utilizó para determinar cortisol salival mediante radioinmunoensayo utilizando un kit comercial (DIAsource InmunoAssays SA, Louvain-la-Neuve, Bélgica). La sensibilidad del método es $0,5 \mathrm{ng} / \mathrm{ml}$ y los coeficientes de variación intra e interensayo son $5,5 \%$ y $10,6 \%$ respectivamente. Valor de referencia: $1,2-7,5 \mathrm{ng} / \mathrm{ml}$.

Dado la variabilidad conocida de la secreción de cortisol, se utilizó el promedio del resultado de dos muestras obtenidas de cortisol salival del mismo sujeto, en días distintos, pero en el mismo rango horario.

\section{Estado nutricional del lactante}

Se registró el peso en kilogramos mediante una pesa previamente calibrada y la talla en centímetros mediante un estadiómetro portátil. La evaluación nutricional se realizó mediante indicadores $\mathrm{P} / \mathrm{E}, \mathrm{P} / \mathrm{T}$ y T/E de acuerdo a tablas de referencia de la OMS, clasificando el estado nutricional según puntos de corte internacional ${ }^{32,33}$. 


\section{Tamaño muestral}

Dado que en el estudio se evaluaron todos los lactantes que estaban en ese momento viviendo con sus madres en el CPF y no existen publicaciones nacionales que hagan referencia a concentración de cortisol salival en lactantes chilenos, no se realizó cálculo de tamaño muestral.

\section{Análisis estadístico}

Se informaron los datos descriptivos mediante tablas de frecuencia absolutas y relativas para las variables cualitativas, $y$ las variables cuantitativas fueron expresadas a través de mediana y rango intercuartil (IQR) o media y desviación estándar según su distribución. Se utilizaron los test de Fisher y Mann-Withney para evaluar significancia de la asociación, considerando significativo un $\mathrm{p}<0,05$. Los datos fueron procesados y analizados utilizando el Programa STATA 15.

\section{Comité ético}

Este estudio fue autorizado por la Unidad Técnica de Dirección Regional Metropolitana de Gendarmería de Chile y aprobado por el Comité de Ética del Servicio de Salud Metropolitano Central. Cada madre firmó un consentimiento informado previo a la aplicación del estudio.

\section{Resultados}

Entre agosto y diciembre del 2017 se reclutaron a 48 niños entre 1 y 24 meses. Ingresaron al grupo caso
13 lactantes ( 1 abandonó el estudio) y 30 al grupo control. Todos los niños que ingresaron al estudio eran chilenos, solo una madre era de nacionalidad peruana. La mediana de la edad cronológica de los niños fue de 7 meses (IQR 5-11 meses). En el 90,5\% de los niños la edad gestacional fue mayor o igual a 37 semanas. Hubo predominio del sexo masculino. Todos los niños del $\mathrm{CPF}$ recibieron en algún momento lactancia materna y solo lo hicieron casi la mitad de los niños del CESFAM (100\% vs $45,2 \%$ respectivamente, $\mathrm{p}=0,01$ ) (tabla 1 ). En cuanto a la evaluación nutricional de los niños el $71,4 \%$ eran eutróficos, $23,7 \%$ tenían malnutrición por exceso y solo un niño tuvo riesgo desnutrición. No encontramos diferencias cuando se analizó el estado nutricional según lugar.

Aunque todas las madres sabían leer y escribir, el nivel educacional alcanzado fue menor entre las madres del CPF ( $p=0,03$ ). Por otro lado, el 30\% de madres tuvieron alguna patología durante su gestación siendo significativamente más frecuente $(58 \%)$ en las madres del CPF ( $\mathrm{p}=0,01)$; las patologías más frecuentes fueron síndrome hipertensivo del embarazo $(9,5 \%)$ y diabetes gestacional (9,5\%) (tabla 2).

La mediana del cortisol salival en los niños de la muestra fue de 2,1 ng/ml (IQR 1,2 a 1,8), sin diferencias significativas entre los niños del CPF y los del CESFAM (2,3 ng/ml IQR 1,1 a 2,7 vs 2,1 IQR 1,6 a 2,9, respectivamente). Con respecto al cortisol materno la mediana fue de $3,8 \mathrm{ng} / \mathrm{ml}$ (IQR 3,0 a 5,3) sin diferencia significativa entre las madres privadas de libertad y las del CESFAM (tabla 3).

Tabla 1. Características demográficas de los niños según lugar

\begin{tabular}{|c|c|c|c|c|c|c|}
\hline & \multicolumn{2}{|c|}{$\mathrm{CPF}(n=12)$} & \multicolumn{2}{|c|}{ CESFAM $(n=30)$} & \multicolumn{2}{|c|}{ Total $(n=42)$} \\
\hline & Mediana & IQR & Mediana & IQR & Mediana & IQR \\
\hline \multirow[t]{2}{*}{ Edad (meses) } & 7 & $7-11$ & 6 & $4-15$ & 7 & $5-11$ \\
\hline & Media & DS & Media & DS & Media & DS \\
\hline Peso/edad (z) & 0,2 & 0,6 & 0,4 & 0,7 & 0,3 & 0,7 \\
\hline Talla/edad (z) & $-0,9$ & 1,0 & $-0,3$ & 1,0 & $-0,4$ & 1,0 \\
\hline \multirow[t]{2}{*}{ Peso/talla (z) } & 0,9 & 1,0 & 0,8 & 0,9 & 0,8 & 0,9 \\
\hline & $n$ & $\%$ & $n$ & $\%$ & $n$ & $\%$ \\
\hline \multicolumn{7}{|l|}{ Edad gestacional } \\
\hline$<37$ semanas & 1 & 2,3 & 3 & 7,1 & 4 & 9,5 \\
\hline$\geq 37$ semanas & 11 & 26,1 & 27 & 64,2 & 38 & 90,5 \\
\hline \multicolumn{7}{|l|}{ Sexo } \\
\hline Masculino & 8 & 15,3 & 14 & 33,3 & 22 & 52,0 \\
\hline Femenino & 4 & 9,5 & 16 & 38,0 & 20 & 48,0 \\
\hline LM presente & 12 & 28,5 & 19,0 & 45,2 & 31 & 73,8 \\
\hline
\end{tabular}

$\mathrm{n}=$ tamaño muestral, $\%$ = porcentaje del tamaño muestral total; CPF: centro penitenciario, CESFAM: centro de salud familiar; LM = lactancia materna. 
Tabla 2. Estado nutricional de los niños según lugar

\begin{tabular}{|c|c|c|c|c|c|c|c|}
\hline & \multicolumn{2}{|c|}{ CPF } & \multicolumn{2}{|c|}{ CESFAM } & \multicolumn{2}{|c|}{ Total } & \multirow[t]{2}{*}{$p$} \\
\hline & $\begin{array}{c}n=12 \\
n\end{array}$ & $\begin{array}{c}(28 \%) \\
\%\end{array}$ & $\begin{array}{c}n=30 \\
n\end{array}$ & $\begin{array}{c}(71 \%) \\
\%\end{array}$ & $\begin{array}{c}n=42 \\
n\end{array}$ & $\begin{array}{c}(100 \%) \\
\%\end{array}$ & \\
\hline Evaluación nutricional actual & & & & & & & 0,72 \\
\hline Desnutrición & 0 & - & 0 & - & 0 & - & \\
\hline Riesgo de desnutrición & 0 & - & 1 & 3,3 & 1 & 2,3 & \\
\hline Eutrófico & 10 & 83,3 & 21 & 70,0 & 31 & 71,4 & \\
\hline Sobrepeso & 1 & 8,3 & 6 & 20,0 & 7 & 16,6 & \\
\hline Obeso & 1 & 8,3 & 2 & 6,6 & 3 & 7,1 & \\
\hline
\end{tabular}

CPF: centro penitenciario, CESFAM: centro de salud familiar

Tabla 3. Características demográficas de las madres según lugar

\begin{tabular}{|c|c|c|c|c|c|c|}
\hline & \multicolumn{2}{|c|}{ CPF $n=12$} & \multicolumn{2}{|c|}{ CESFAM $n=30$} & \multicolumn{2}{|c|}{ Total $n=42$} \\
\hline & Mediana & IQR & Mediana & IQR & Mediana & IQR \\
\hline Edad (años) & 24,0 & $25-33$ & 25,5 & $23-30$ & 26,0 & $24-30$ \\
\hline \multicolumn{7}{|c|}{ Nivel escolaridad completada* (n y \%) } \\
\hline Ninguno & 4 & 9,5 & 3 & 7,1 & 7 & 16,6 \\
\hline Basica & 6 & 14,2 & 4 & 9,5 & 10 & 23,8 \\
\hline Media & 2 & 4,7 & 19 & 45,2 & 21 & 50,0 \\
\hline Superior & 0 & 0 & 4 & 9,5 & 4 & 9,5 \\
\hline \multicolumn{7}{|c|}{ Enfermedad embarazo* } \\
\hline Presente & 7 & 16,6 & 6 & 14,2 & 13 & 30,9 \\
\hline
\end{tabular}

$\mathrm{n}=$ tamaño muestral, \% = porcentaje del tamaño muestral; CPF: centro penitenciario, CESFAM: centro de salud familiar, *(test Fisher's $p<0,05)$.

Tabla 4. Concentración de cortisol salival según lugar

\begin{tabular}{|c|c|c|c|c|c|c|}
\hline & \multicolumn{2}{|c|}{ CPF $n=12$} & \multicolumn{2}{|c|}{ CESFAM $\quad n=30$} & \multicolumn{2}{|c|}{ Total $n=42$} \\
\hline & Mediana & IQR & Mediana & IQR & Mediana & IQR \\
\hline \multicolumn{7}{|c|}{ Cortisol salival ng/ml } \\
\hline Niños & 2,3 & 1,1 a 2,7 & 2,1 & 1,6 a 2,9 & 2,1 & 1,2 a 1,8 \\
\hline Madres & 4,6 & 3,8 a 7,3 & 3,7 & 2,4 a 4,7 & 3,8 & 3,0 a 5,3 \\
\hline
\end{tabular}

CPF: centro penitenciario, CESFAM: centro de salud familiar IQR: rango intercuartil.

Luego de medir el DSM mediante el ASQ3, observamos que 13 niños tuvieron déficit de DSM, 61\% de los cuales tuvieron más de un dominio alterado siendo las áreas de mayor riesgo la motora fina, gruesa y de resolución de problemas. Al realizar el análisis de DSM, según lugar de residencia del niño, encontramos que el déficit del DSM fue más frecuente entre los niños que vivían fuera del $\mathrm{CPF}$ con un $2,3 \%$ vs $28,5 \%$ respectivamente (Test de Fisher $\mathrm{p}=0,06)$ (tabla 4). Del total de niños de la muestra, cuatro niños eran menores de 37 semanas y sólo uno de estos tuvo déficit de DSM.

Al comparar la concentración de cortisol de los niños de acuerdo al DSM y los valores de cortisol de las madres con el DSM, no se encontró asociación significativa (tabla 5). Tampoco encontramos diferencias al evaluar la relación entre el DSM del niño y nivel educacional materno alcanzado, independientemente del lugar de residencia del niño. 
Tabla 5. DSM de los niños según lugar

\begin{tabular}{|c|c|c|c|c|c|c|c|}
\hline & \multicolumn{2}{|c|}{ CPF } & \multicolumn{2}{|c|}{ CESFAM } & \multicolumn{2}{|c|}{ Total } & \multirow[t]{2}{*}{$p$} \\
\hline & $\begin{array}{c}n=12 \\
n\end{array}$ & $\begin{array}{c}(28 \%) \\
\%\end{array}$ & $\begin{array}{c}n=30 \\
n\end{array}$ & $\begin{array}{c}(71 \%) \\
\%\end{array}$ & $\begin{array}{c}n=42 \\
n\end{array}$ & $\begin{array}{c}(100 \%) \\
\%\end{array}$ & \\
\hline DSM & & & & & & & 0,06 \\
\hline Normal & 11 & 26,1 & 18 & 42,8 & 29 & 69 & \\
\hline Deficiente & 1 & 2,3 & 12 & 28,5 & 13 & 30,9 & \\
\hline
\end{tabular}

CPF: centro penitenciario, CESFAM: centro de salud familiar, DSM: desarrollo psicomotor.

\section{Discusión}

El principal hallazgo de este estudio es que los niños que viven con sus madres en el CPF tienen concentraciones de cortisol salival similares que los que se atendieron en el CESFAM. Además, nos parece importante resaltar que el déficit del DSM fue llamativamente más frecuente en los niños del CESFAM, aunque la diferencia no fue significativa $(\mathrm{p}=0,06)$ es posible que esto se deba al tamaño muestral.

Los resultados del único estudio realizado en una población similar a la nuestra difieren un poco de los encontrados por nosotros. Morales M, encontró en una muestra de niños obtenidos del mismo CPF $(\mathrm{n}=15)$, luego de evaluar el DSM mediante EEDP, que el $25 \%$ de los niños tenían DSM alterado ${ }^{18}$. Desde la época en que se publicó el trabajo mencionado han existido cambios, como la creación de la sala cuna, que podrían ser responsables de las diferencias encontradas. Creemos que el contacto de los niños con sus madres y la estimulación recibida por estos en el jardín infantil del CPF, podría influir en los resultados observados. Nos parece importante resaltar que el jardín infantil mencionado cuenta, además del personal habitual en este tipo de establecimientos, con la presencia de una psicomotricista y consulta psicológica semanal lo que podría potenciar el impacto del cuidado materno. También nos llama la atención la alta frecuencia de lactancia materna en los niños del CFP (100\%) la cual podría estar favorecida porque los niños duermen junto a sus madres, situación promovida por el programa gubernamental "Creciendo juntos" ${ }^{34}$. Respecto al impacto de la lactancia materna sobre el DSM, Chui y col, han demostrado que existe una asociación lineal tipo "dosis respuesta" entre la duración de la LM y el desarrollo motor y cognitivo ${ }^{35}$. Por otro lado, la frecuencia de déficit de DSM que observamos en los niños atendidos en el CESFAM (28\%\%) es similar a la encontrada en estudios nacionales $(30 \%)^{36}$.

La concentración de cortisol salival detectada en ambos grupos de niños del estudio se encuentra dentro del rango de normalidad. Tollenaar y col, analiza- ron la concentración de cortisol salival matinal en una cohorte de 300 lactantes mediante mediciones a las 6 semanas, a los 5 y a los 10-12 meses de vida mostrando que los rangos normales en lactantes menores de un año se encuentran entre 1,59 y $9,05 \mathrm{ng} / \mathrm{ml}^{37}$. Otro estudio realizado por Albers y cols, midieron cortisol salival a 64 lactantes menores de un año en una sala cuna, determinando que las concentraciones normales se encontraban entre 1,92 y $4,34 \mathrm{ng} / \mathrm{ml}^{12}$.

El cortisol, en condiciones normales, muestra un marcado ritmo circadiano, en adultos y niños. Los niveles de cortisol son más altos en la mañana, seguido de un descenso abrupto inmediato después del desper$\operatorname{tar}^{38-41}$. Se cree que los lactantes desarrollan este ritmo circadiano del cortisol durante el primer año de vida, pero exactamente cuándo, aún no se ha establecido. No existe claridad respecto a la variabilidad de la concentración de esta hormona cuando se mide de forma repetida. Un estudio pionero de Ivars et al, describen que la mayoría de los lactantes comienzan su ritmo circadiano al cuarto mes de edad, sin embargo, describen una importante variabilidad individual de estos niveles, tal como se muestra en nuestro estudio ${ }^{42}$. Se sabe que la concentración de cortisol varía ampliamente en los mismos sujetos a distintas horas del día y de acuerdo a la edad ${ }^{40}$. Otros factores, dependientes de la estandarización de la toma de muestra, también podrían explicar la variación mencionada. En nuestro estudio el período de ayuno necesario, previo a la toma de la muestra $(1 \mathrm{~h})^{43}$, fue evaluado mediante cuestionario, lo que también podría explicar parte de la variabilidad observada.

Respecto a las limitaciones de nuestro estudio, la escasa cantidad de niños evaluados en el CPF es uno de los factores que podría explicar la falta de diferencias significativas encontradas en nuestro trabajo. Otro aspecto a considerar es que el diseño del estudio que no permite realizar asociaciones de causalidad. En un próximo estudio debería considerarse un grupo control de niños que vivan con sus madres en una penitenciaria sin jardín infantil. Creemos que también es necesario realizar el seguimiento de los niños evaluados para explorar si el DSM y estrés se afectan a largo plazo. 
Cabe destacar que es el primer estudio en Chile que mide el nivel de estrés de madres e hijos en un centro penitenciario, que incluye una guardería interdisciplinaria, y su asociación con el DSM que existe en estos niños que viven bajo estas condiciones.

Como conclusión, creemos que nuestro estudio es un aporte que debería ser considerado al momento de evaluar el beneficio de la convivencia de los lactantes con sus madres en centros penitenciarios del país, teniendo la obligación de respetar los derechos de los niños.

\section{Responsabilidades Éticas}

Protección de personas y animales: Los autores declaran que los procedimientos seguidos se conformaron a las normas éticas del comité de experimentación humana responsable y de acuerdo con la Asociación Médica Mundial y la Declaración de Helsinki.

Confidencialidad de los datos: Los autores declaran que han seguido los protocolos de su centro de trabajo sobre la publicación de datos de pacientes.

\section{Derecho a la privacidad y consentimiento informa-} do: Los autores han obtenido el consentimiento informado de los pacientes y/o sujetos referidos en el artículo. Este documento obra en poder del autor de correspondencia.

\section{Conflicto de intereses}

Los autores declaran no tener conflicto de intereses.

\section{Agradecimientos}

Agradecemos a las madres de los niños y las niñas que aceptaron participar de nuestro estudio, a las profesionales de la unidad técnica de región metropolitana de gendarmería de Chile, al equipo de gendarmería de CPF San Joaquín, a las personas que trabajan en el jardín infantil , a la psicóloga a cargo, y al equipo directivo administrativo de CESFAM Padre Vicente Irarrázaval.

\section{Referencias}

1. Schonhaut L, Álvarez J, Salinas P. El pediatra y la evaluación del desarrollo psicomotor. Rev Chil Pediatr. 2008;79:2631.

2. Subsecretaría de Salud Pública División de Planificación Sanitaria: II Encuesta de Calidad de Vida y Salud, Chile 2006 http://www.crececontigo.gob.cl/wpcontent/uploads/2015/11/ENCAVI-2006. pdf. Última visita: noviembre de 2017.

3. Committee of children with disabilities: Developmental surveillance and screening of infant and young children. Pediatrics. 2001;108:192-5.

4. Registros estadísticos mensuales, DEISMINSAL 2016 https://reportesdeis.minsal. cl/REM/2016/REM03SECCIONA2/ REM03SECCIONA2.aspx. Última visita: noviembre de 2018.

5. Schonhaut L, Armijo I, Schonstedt M, Alvarez J, Cordero M. Validity of the ages and stages questionnaires in full term and preterm infants. Pediatrics 2013;131:e1468-75.

6. Armijo I, Schonhaut L, Cordero M. Validation of the Chilean version of the Ages and Stages questionnaire (ASQ-CL) in community health settings. Early human development, 2015;91:12:671-6.

7. Zijlmans M, Riksen-Walraven M, De Weerth C. Associations between maternal prenatal cortisol concentrations and child outcomes: A systematic Review Neuroscience and Biobehavioral Reviews. 2015;53:1-24.

8. McLaughlin K, Sheridan M, Tibu F, et al. Causal effects of the early caregiving environment on development of stress response systems in children. PNAS. 2015; 112;18:5637-42.

9. Rankovi M, Vuji J, Maglajiuki S. Salivary cortisol as a biomarker of stress in mothers and their low birth weight infants and sample collecting challenges. J Med Biochem 2016; 35.

10. Nagakura T, Tanaka T, Arita M, et al. Salivary cortisol monitoring: Determination of reference values in healthy children and application in asthmatic children. Allergy and Asthma. 2012;33:362-9.

11. Finegood E, Wyman C, O'Connor T, et al. Salivary Cortisol and Cognitive Development in Infants from LowIncome Communities. Stress, 2017.

12. Albers E, Beijers R, Riksen-Walraven M, et al. Cortisol levels of infants in center care across the first year of life: links with quality of care and infant temperament. Stress. 2016;19(1):8-17.

13. Panagiota D, Letourneau N. Approaches to Salivary Cortisol Collection and Analysis in Infants. Biological Research for Nursing 2014;16:398-408.

14. Forns J, Vegas O, Julvez J, et al. Association between Child Cortisol Levels in Saliva and Neuropsychological
Development during the Second Year of Life. Stress Health. 2013;30:142-8.

15. Lovell B, Moss M, Wetherell M. Perceived stress, common health complaints and diurnal patterns of cortisol secretion in young, otherwise healthy individuals, Hormones and Behavior. 2011;60:301-5.

16. Beijers R, Riksen-Walraven J, De Weerth M. Cortisol regulation in 12-month-old human infants: Associations with the infants' early history of breastfeeding and co-sleeping. Stress. 2013;16:267-77.

17. Valenzuela E, Marcazzolo X, Stuven A, et al. Impacto social de la prisión Femenina en Chile, Propuestas para Chile Concurso Políticas Públicas, 2012. http:// www.minjusticia.gob.cl/media/2013/04/ presentacion-impacto-social.pdf. Última visita: diciembre de 2017.

18. Morales M, Núñez P. “Desarrollo Psicomotor y Procesamiento Sensorial de menores de 4 a 18 meses de edad, hijos de internas del Centro Penitenciario Femenino Santiago". Tesis Entregada a la universidad de chile para obtención de grado de licenciatura en kinesiología, 2011 "observaciones no publicadas".

19. Lejarraga H, Berardi C, Ortale S. et al. Growth, development, social integration and parenting practices on children living with their mothers in prison. Arch Argent Pediatr. 2011;109:485-91.

20. Sroufe A, Egeland B, Carlson E, Collins, A. The development of the person: The Minnesota Study of Risk and Adaptation 
from Birth to Adulthood. J Can Acad Child Adolesc Psychiatry 2007;16:4

21. Murray J, Murray L. Parental incarceration, attachment, and child psychopathology. Attachment \& Human Development 2010;12(4):289-309.

22. Phillips S., Harm N. Women prisoners: A contextual framework. Women and Therapy. 1997;20:1-9.

23. Robertson O. The impact of parental imprisonment on children. Women in Prison and Children of Imprisoned Mothers Series. Quaker United Nations Office. 2008 editorial, The University of Chicago. 2008;1-70.

24. Cho R. Understanding the mechanism behind maternal imprisonmentand adolescent school dropout. Family Relations 2011;60(3):272-89.

25. Gendarmería de Chile. Estadísticas de información penitenciaria. Disponible http://www.gendarmeria.gob.cl/. Última visita: octubre de 2017.

26. Orientaciones Técnicas Específicas. Modalidad Residencias de Protección para Lactantes de Madres Internas en Recintos Penitenciarios disponible en la web, http://www.sename.cl/wsename/otros/ proteccion/Residencias\%20Lactantes $\% 20$ Madres\%20Recluidas.pdf. Último visita julio de 2017.

27. Estudio de sistematización del diseño e implementación del piloto línea materno infantil-Programa Abriendo Caminos, Subsecretaria de redes sociales, Ministerios de desarrollo social, gobierno de Chile. 2015. Disponible en la web: http://www.ministeriodesarrollosocial. gob.cl/btca/txtcompleto/Final_Piloto_ Materno_Infantil.pdf. Última visita: enero de 2018.

28. Valenzuela E, Marcazzolo X, Stuven A, Larroulet $\mathrm{P}$, et al. Impacto social de la prisión Femenina en Chile, Propuestas para Chile Concurso Políticas Públicas. 2012 disponible en la web, http://www. minjusticia.gob.cl/media/2013/04/ presentacion-impacto-social.pdf. Última visita: julio de 2017.

29. Schonhaut L, Armijo I. Aplicabilidad del Ages \& Stages Questionnaires para el tamizaje del desarrollo psicomotor. Rev Chil Pediatr 2014;85(1):12-21.

30. Schonhaut L, Salinas P, Armijo I, Schönstedt M, Álvarez J, Manríquez M. Validación de un Cuestionario Autoadministrado para la Evaluación del Desarrollo Psicomotor. Rev Chil Pediatr. 2009;80(6):513-9.

31. Garrido M, Romero C, Cid M. Detección de cortisol salival nocturno en una muestra de sujetos de Santiago de Chile, mediante la técnica de electroquimioluminicencia. Rev Chil Endocrinol Diabetes. 2015;8:102-7.

32. Norma técnica para la supervisión de niños y niñas de 0 a 9 años en la Atención Primaria de Salud. Programa Nacional de Salud de la Infancia. Ministerio de Salud Gobierno de Chile. 2014. Disponible en la web, http://www:2014_Norma Técnica para la supervisión de niños y niñas de 0 a 9 en APS_web(1).pdf.Última visita: julio de 2017.

33. Referencias OMS para evaluación antropométrica de niños y niñas menores de 6 años. Organización Panamericana de salud. Ministerio de Salud Gobierno de Chile. 2014.Disponible en la web, http://web.minsal.cl/sites/default/files/ files/2013_Referencia\%20OMS\%20 para $\% 201 \mathrm{l} \% 20$ evaluaci $\%$ C3\%B3n $\% 20$ antropom $\% \mathrm{C} 3 \%$ A 9 trica $\% 20$ menores $\% 20$ de $\% 206 \% 20 a \%$ C3\%B1os.pdf. Última visita: diciembre de 2017.

34. Informe Programas de Reinserción. Gerdarmería, Ministerio de Justicia, Chile. Marzo 2017. Requisitos y Antecedentes para acceder al Programa Creciendo Juntos. Disponible en la web: https://html gendarmeria.gob.cl/doc/transparencia/ ley20285/doc_2009/sub_beneficios/
doc/2017/Requisitos_Programa Creciendo_Juntos_(ex_PAMEHL).pdf Última visita: enero de 2018.

35. Chiu W, Liao H, Chang P. Duration of breast feeding and risk of developmental delay in Taiwanese children: a nationwide birth cohort study. Pediatr Perinat Epidemiol. 2011;25:519-27.

36. Bedregal P. Hacia la renovación en las políticas de infancia en Chile. Rev. chil. pediatr. 2014;85:7-11.

37. Tollenaar M, Jansen J, Beijers R, RiksenWalraven J, Weerth C. Cortisol in the first year of life: Normative values and intra-individual variability Early Human Development 2010;86:13-6.

38. Rolfsjord L, Bakkeheim E, Løvold T, et al. Morning Salivary Cortisol in Young Children: Reference Values and the Effects of Age, Sex, and Acute Bronchiolitis. The Journal of Pediatrics. 2017;184:193-8.

39. Flom M, St. John A, Meyer J, Tarullo A. Infant hair cortisol: associations with salivary cortisol and environmental context Dev Psychobiol 2016;9999:1-13.

40. Cordero M, Aguilar A, Sánchez M, et al. Cortisol salival como indicador de estrés fisiológico en niños y adultos; revisión sistemática, Nutr Hosp. 2014;29:960-8.

41. Camacho E, Michel C, Santos O. Niveles de cortisol y estilo de vida en estudiantes universitarios sanos de México y España, Revista Latinoamericana de Medicina Conductual 2011;1:22.

42. Ivars K, Nelson N, Theodorsson A, Theodorsson E, Strom J, Morelius E. Development of Salivary Cortisol Circadian Rhythm and Reference Intervals in Full-Term Infants.PLoS ONE 2015;12:8.

43. Garrido M, Romero C, Cid M. Detección de cortisol salival nocturno en una muestra de sujetos de Santiago de Chile, mediante la técnica de electroquimioluminicencia. Rev. Chil. Endocrinol. Diabetes. 2015; 8:102-7. 